\title{
Review
}

Ting Miao*

\section{Common types of infection with multitypes of HPV on uterine cervix}

DOI: $10.2478 / \mathrm{ii}-2018-0013$

Received February 21, 2018; accepted February 21, 2018; published online May 15, 2018

\begin{abstract}
Persistent infection by human papillomavirus (HPV) is an important factor causing cervical cancer. In recent years, infection with multiple HPV types has been confirmed in various studies. High-risk HPV 16 and 18 and low-risk HPV 6 are the most common causes of multitype HPV infection. Infection with multiple types of HPV, which results from individual susceptibility, is crucial in tumor susceptibility. This paper summarizes the common types of multiple HPV infection to enable further research on the relationship between HPV and tumor susceptibility.
\end{abstract}

Keywords: HPV, infection, cervical lesions, cervical cancer

\section{Introduction}

Cervical cancer (CC) occurs through the combined actions of multiple factors, steps, and genes. However, in recent years, persistent infection by human papillomavirus (HPV) has been shown to be a key factor causing CC. Therefore, screening for cervical HPV infection to prevent CC has become the consensus of international agency for cancer prevention [1]. Multitype infection refers to the infection of the cervical epithelium by multiple HPV types or the detection of these types in various cervical lesions. In recent years, HPV coinfection in the cervix has been confirmed by an increasing number of tests. Therefore, increasing attention must be given to multitype infections. Many problems are associated with this phenomenon. This paper aims to summarize the common types of multiple HPV infections in different populations to enable further research on this aspect.

\section{Common types of multitype HPV infection in the nonspecific population}

Here, nonspecific population refers to all the women subjected to HPV detection in a certain area in accordance with the requirements of age, regardless of their anthropological characteristics, cytological status of cervical exfoliative cells, or histological status of their cervical epithelium.

\subsection{Common types of multitype HPV infection in the population under investigation}

Fiqueiredo Alves et al. [2] investigated the HPV infection types in 432 unimmunized female adolescents from Goiania, Brazil, by using polymerase chain reaction (PCR) and the reverse dot-blot technique. A total of 30 HPV types, including 19 high-risk types and 11 low-risk types, were evaluated. The subjects were aged 15-19 years (average: $17.2 \pm 1.3$ years). Results showed a HPV infection rate of 28\% (121/432). A total of 54 cases presented multitype infection (54/432, 12.5\%), which accounted for $44.6 \%$ of the infected cases (54/121).

Department of Pharmacy, Tianjin Fourth Center Hospital, Tianjin, China

*Correspondence: Ting Miao, E-mail: tingmiao82@163.com 
The most common types of HPV infection were types 16, 51, 31, 52, and 18. By contrast, the most common types of multitype infection were types $16,51,18,31$, and 52. The most common type of HPV infection in both single and multiple infections was type 16, and the most low-risk infection type was HPV type 6 [2].

Menegazzi et al. [3] detected HPV in the genital tract specimens of 654 volunteer women from the south of Italia by means of PCR, and type-detected HPV-positive specimens by using INNO-LiPA HPV Genotyping System. Cytological examination was conducted on the specimens simultaneously. A total of 29 types were detected; these types included HPV 6, 11, 16, 18, 26, 31, 33, 35, 39, 40, 42, 43, 44, 45, 51, 52, 53, 54, 56, 58, 59, 66, 68, 69, 71, 70, 73, 74, and 82. Among these types, HPV 16, 18, 26, 31, 33, 35, 39, 45, 51, 52, 53, 56, 58, 59, $66,68,73$, and 82 were of high risk. Results showed that 300 cases were positive for HPV DNA; this number accounted for $45.9 \%$ of the population being tested. Additionally, 125 cases presented multiple infections and accounted for $41.7 \%$ of the detected population. Among the people testing positive for HPV, 267 cases (89.0\%) were of high-risk HPV infection. With increased age, HPV and multiple infections decreased. At the age of $\leq 40$ years, the detection rate was $56.1 \%$; at $>40$ years, the detection rate was $37.7 \%(P<0.0001)$. In single and multiple infections, regardless of the presence or absence of cytological abnormality, HPV 6 and 16 were the most common types [3]. This result is similar to those of Fiqueiredo Alves et al. [2].

Vaccarella et al. [4] performed PCR typing on the reproductive swab cell samples of 31717 women in the south of Sweden. A total of 16 types (HPV 6, 11, 16, 18, 31, 33, 35, 39, 45, 51, 52, 56, 58, 59, 66, and 68) were detected. The average age of the subjects was 25.1 years (interquartile range: 19-29 years). Approximately $38.5 \%$ (12211/31717) of the samples were HPV positive. Among these 12211 positive samples, the detection rate of multitype infection was 41.9\% (5120/12211). A total of 7091 cases displayed single HPV infection (7091/31717, 22.4\%), 3246 cases showed dual infection (3246/31717, 10.2\%), and 1874 cases exhibited more than two types of HPV $(1874 / 31717,5.9 \%)$. In total, the detection rates were 10.2\%, 6.1\%, 5.4\%, 5.2\%, 5.2\%, and 4.8\% for HPV 16, 51, 31, 18,66 , and 52, respectively [4]. The detection rate of HPV 16 was relatively high among the multitype infections.

Several studies on HPV infection investigated the sexual behavior of women. Chaturvedi et al. [5] assessed 25 HPV types in the exfoliative cells of sexually active women in Costa Rica. Results showed that among the individuals who satisfied the study requirements and were detected with the virus, 50.0\% (2938/5871) were HPV positive, and $42.3 \%$ (2479/5871) presented with at least one of the 25 infection types. The subjects with multitype infection accounted for 18.2\% (1070/5871). However, the proportion of multitype infection by oncogenic HPV types was relatively high $(33.9 \%, 673 / 1983)$. The infection rate of multiple types in the $\alpha 9$ population (HPV 16, 31, 33, 35, 52, and 58) was $19.4 \%$ (241/1245), but that of HPV 16 was considerably high (65.2\%; 18/488). Additionally, the infection rate of multiple types among the $\alpha 7$ population (HPV 18, 39, 45, 59, and 70) was substantially low at 9.8\% (71/721), but that of HPV 18 was remarkably high at 70.7\% (133/188). HPV 54 (48.6\%) and 34 (86.7\%) were recorded with higher multitype infection rates than the other types in this population [5]. The reason why HPV 16 and 18 are highly significantly more prevalent in multitype infection than the other types in the same population remains unknown.

Similar studies were conducted in China. Gu et al. [6] detected HPV in the cervixes of 12226 women aged 35-59 years from five community health centers in Wuxi New Region. The HPV types (14 high-risk types and 2 low-risk types) were identified through gene sequencing technology. The total HPV infection rate in 12226 women was $12.15 \%$ (1486/12226). The HPV single and multiple infection rates were 10.12\% (1237/12226) and 2.04\% (249/12226), respectively. Multiple infection included double, triple, fourfold, and fivefold infections, with infection rates of 1.60\% (196/12226), 0.34\% (41/12226), 0.08\% (10/12226), and 0.02\% (2/12226), respectively. HPV 16 combined with another high-risk type was the most common high-risk combination (32.53\%, 81/249), with combinations involving types 35 (10.04\%, 25/249), 39 (8.43\%, 21/249), and 18 (8.03\%, 20/249) following the rank. In high risk-low risk combinations, HPV 6 exhibited the highest detection rate $(9.23 \%$, 23/249) [6]. HPV 16 and 6 recorded considerably high detection rates in the high-risk and low-risk types.

\subsection{Common types of multitype HPV infection in the outpatient population}

Herein, the term "outpatient subjects" refers to the women who were tested for HPV infection due to various gynecologic causes. Dickson et al. [7] adopted the PCR typing technique to identify and statistically analyze 
the HPV infection type in liquid-based cervical cytology specimens of 309471 American women; these women were aged 18-65 years (average: $39.2 \pm 12.1$ years) and were subjected to Pap smear test and vaginoscopy; their cell specimens were collected from July 2007 to May 2011. Data were obtained from 47 laboratories. Results showed that 74543 cases (24.1\%) were infected with at least one HPV type, and 65492 cases $(21.1 \%)$ were infected with one of the most popular $24 \mathrm{HPV}$ types $(6,11,16,18,31,33,35,45,52,53,54,58,59,61,62,66,68,70$, $72,81,82,83,84$, and 89$)$. A total of 14181 cases were infected with two or more HPV types, which accounted for $4.6 \%$ of all the specimens and $19.0 \%$ of the positive specimens. Among these cases, 12016 (3.9\%), 1936 $(0.6 \%)$, and $229(0.1 \%)$ cases were infected with two, three, and four types of HPV, respectively. The most common multitype infections were types $16,53,52,66$, and 31 . The most common HPV type was HPV 16, which accounted for $4.1 \%$ of all the specimens. The proportion of its involvement in multitype infection was $1.2 \%$, which accounted for $26.2 \%$ of the detection rate in multitype infection [7]. Bayram et al. [8] tested the liquid-based cervical cytology specimens of 520 women of childbearing age in the Obstetrics and Gynecology Clinic of the Medical College of Turkey, Gaziantep University. HPV DNA was detected by nested PCR and typed by means of reverse dot-blot genotyping. A total of 29 types were identified, of which 11 were low-risk types, three were potential high-risk types (HPV 26, 53, and 66), and 15 were high-risk types. The HPV detection rate was $16.9 \%$ (88/520), of which $65.9 \%$ (58/88), 27.3\% (24/88), and 6.8\% (6/88), corresponded to single infection, dual infection, and infection by more than two types, respectively. Multitype infection accounted for $34.1 \%$ of the total detection rate. The infection caused by HPV 16 and 18 combination accounted for $20 \%$ of the multitype infections (6/30). The combination of HPV 58 and 16 or 18 accounted for $10 \%$ of multitype infections, which was more than that of the combined infection by other types. HPV 6 was the most common low-risk type causing multitype infections $(n=8,26.7 \%)$ [8]. HPV 16 and 18 among the high-risk types and HPV 6 among the low-risk types showed relatively high detection rates. Similar studies were conducted in China. Zhang et al. [9] applied PCR in vitro amplification and membrane hybridization for the HPV typing of 10764 women who visited the Gynecologic Clinic of Hainan Provincial People’s Hospital. Among 10764 specimens, the positive rate was $19.38 \%$ (2811/10764). The multiple-infection rate was 4.96\% (534/10764), which accounted for $19.0 \%$ of the positive detection rate (534/2811). Statistical analysis on multiple infection revealed that infection with at the most six different HPV subtypes was detected in 534 cases with multitype infection. In double and triple infections, types 52 and 58 displayed the highest detection rates, but type 16 showed the highest detection in fourfold infection. Moreover, types 58, CP8304, and type 53 were the most common infectious agents. The trend of HPV infection with different subtypes detected from fivefold and sixfold infections was insignificant [9]. Although different conclusions were obtained from the above-mentioned studies, type 16 generally exhibits high proportions in multiple infections.

Various studies were also conducted in foreign countries. Mollers et al. [10] utilized SPF10 linear probe detection to study the occurrence and distribution of 25 types of HPV infections among 3874 Holland women in three areas. The subjects were aged 18-24 years (median: 21 years). The study result showed that the total HPV detection rate was $47 \%$, of which $26 \%$ corresponded to that of multitype infection. The most common HPV infection types were types 16,51 , and 52 . The most common single infection types were HPV 54, 42, 16, and 70, and the most common coinfection types were HPV 43, 44, 45, 35, and 11 [10]. Types 16 and 18 were not highly detected in multitype infections.

\section{Common types of multitype HPV infection among patients with cervical lesions}

\subsection{Common types of multitype HPV infection among patients with precancerous lesions in the cervix}

Vidal et al.[11] studied the HPV types in 572 American women of two different races with cervical cytological abnormalities by using liquid-based thin-layer cervical cytology and HPV linear array technology. A total of 
37 HPV types were detected. The total-HPV positive rate was 72\% (494/572), in which multitype HPV infection accounted for $73 \%$. Among the high-risk HPV types, the most common in multitype infections were types 51, 52, 56, 59, and 66 in the non-cervical intraepithelial neoplasia (CIN) group; types 16, 31, 39, 51, 52, and 66 in the CIN1 group; types 16, 18, 39, and 52 in the CIN2/3 group; and types 16, 52, 39, 18, 35, 45, 59, and 66 in the CIN3 group [11]. HPV 16 was the most common type among the multiple types of CIN infection in the cervical lesions of different degrees.

Spinillo et al. [12] investigated the possibility of 681 women with histologically diagnosed CIN lesion being infected with multiple types. The SPF10-LiPA detection kit was used for HPV typing. A total of 15 high-risk HPV and 10 low-risk HPV types were detected. Moreover, 677 cases presented the above-mentioned infection types, accounting for $99.4 \%$ of the detected cases. Among these cases, $72.5 \%$ (491/677) were multitype infections. In multitype infection, HPV 6, 11, 16, 18, 31, 51, and 52 exhibited considerably high coinfection rates. Among these infections, the detection rates were as follows: HPV 6, 93.6\% (118/126); HPV 11, 95.2\% (59/62); HPV 16, 82.4\% (262/318); HPV 18, 91.5\% (108/118); HPV 31, 83.9\% (193/230); HPV 51, 91.2\% (124/136); and HPV 52, 86.7\% (111/128) [12]. HPV 16, 18, and 6 still showed considerably high coinfection rates.

Slightly different from the above studies, Beca et al. [13] investigated the HPV genotype distribution among women with high-grade squamous intraepithelial lesion (HSIL); they conducted HC2-HR DNA typing test on 1394 liquid-based thin-layer cervical cytology specimens collected from 2008 to 2011. The PapilloCheck method was used for specimen identification and typing. The multitype infection rates were as follows: HPV 16, 23.03\%; HPV 51, 7.30\%; HPV 42, 6.74\%; HPV 31, 5.62\%; HPV 58, 5.62\%; HPV 56, 5.62\%; HPV 52, 3.93\%; HPV 33, 3.37\%; HPV 35, 2.81\%; and HPV 18, 1.69\% [13]. HPV 16 was the most common, whereas type 18 was the least prevalent.

Correnti et al. [14] collected specimens from 450 women diagnosed with low-grade squamous intraepithelial lesion (LSIL; 200 cases), HSIL (100 cases), and CC (150 cases) by histopathology from December 2002 to March 2009 in Venezuela. PCR HPV typing analysis was also performed. The HPV positive rates were $68 \%$ (136/200) and 95\% (95/100) for LSIL and HSIL, respectively. The multitype infection rates were 44.2\% (42/95) among the HSIL cases and 42.6\% (58/136) among the LSIL cases. Among the LSIL cases with multitype infection, 25 cases were associated with type 16 or 18 , and these cases accounted for $43.1 \%$ of the multitype detection rate (25/58). Furthermore, 14 cases were associated with type 33, 31, 45, or 52, and they accounted for $24.1 \%$ of the multitype detection rate (14/58); among the HSIL cases with multitype infection, 25 cases were associated with type 16 or 18 , which accounted for $59.5 \%$ of the multitype detection rate (25/42); 11 cases were associated with type $33,31,45$, or 52 , and they accounted for $26.2 \%$ of the multitype detection rate (11/42) [14]. Types 16 and 18 remained the most commonly identified types.

\subsection{Common types of multitype HPV infection among patients with CC}

Denny et al. [15] conducted pathological diagnosis and HPV typing on biopsy specimens from 659 women with CC (167 cases from Ghana, 192 cases from Nigeria, and 300 cases from South Africa). They also evaluated $25 \mathrm{HPV}$ types by using reverse-hybridization technique. A total of 570 cases were diagnosed as CC histologically, and $90.4 \%$ of the cases (515/570) were HPV positive. Among these positive cases, single HPV infection accounted for 86.8\% (447/515), multitype infection accounted for 11.1\% (57/515), and HPV infection by unknown type accounted for the remaining 2.1\% (11/515). The detection rates of multitype infections were as follows: HPV 16, 56.1\%; HPV 18, 38.6\%; HPV 45, 33.3\%; HPV 52, 22.8\%; and HPV 35, 19.3\% [15]. Similarly, HPV 16 and 18 showed relatively high coinfection rates. Sykes et al. [16] studied HPV infection in the cervical specimens of 227 New Zealand women (aged $50.7 \pm 16.7$ years on average) who were diagnosed with invasive cervical cancer (ICC). SPF10-LiPA was used for HPV typing. Histopathological results showed that 159 cases $(70.0 \%)$ were of squamous cell carcinoma, 61 cases (26.9\%) were of glandular cancer, and 7 cases (3.1\%) were of adenosquamous carcinoma. HPV DNA and HPV 16 or 18 were detected in 88.5\% (201/227) and 81.1\% $(163 / 201)$ of the cases. The single infection rate was $93.5 \%$ (188/201), and the multitype infection rate was $5.5 \%$ (11/201). Moreover, $1 \%$ of cases were infected by an unknown type. In the multitype infections, the 
most common types included HPV 16, 18, and 52, with detection rates of $63.6 \%(7 / 11), 45.5 \%$ (5/11), and $18.2 \%$ (2/11), respectively [16]. This result is similar to those of Denny et al. [15]. Types 16 and 18 showed remarkably high detection rates.

Li et al. [17] detected HPV DNA in 190 patients diagnosed with CC IIb-III in Chengdu to analyze the distribution characteristics of the HPV subtypes. The HPV-positive rate was 88.95\% (169/190), and the multiple infections accounted for $28.40 \%$ of the positive patients (48/169). Multiple infections were dominated with double infection, and combined infection of HPV 16 with other subtypes was the most common type [17]. Correnti et al. [14] also examined 150 CC specimens and obtained a HPV-positive rate of 98.7\% (148/150) and a multiple-infection rate of $27 \%$ (40/148). A total of 22 cases were multiple infections with type 16 or 18, which accounted for 55\% of the multitype detections (22/40); additionally, 14 cases were multiple infections with type $33,31,45$, or 52, and they accounted for 35\% of the multitype detections (14/40) [14]. Similarly, the highest relative detection rate was that of multitype infection with type 16 or 18. Li et al. [18] studied 30848 cases with HPV infection of ICC from 243 research groups in 1990-2010 from seven popular areas (Africa, East Asia, West/ Central Asia, Europe, North America, South/Central America, and Oceania); they also carried out typing tests on 30357 cases. Results showed that the HPV infection rate of these specimens was $89.9 \%$. This group also studied 66 groups with 12106 cases of ICC. The HPV-positive rate was 93.4\% (11307/12106), and the single- and multitype-infection rates were $80.7 \%(9766 / 12106)$ and 12.7\% (1541/12106), respectively. The most common types of multitype infection were HPV 16 and 18, which accounted for 61.3\% (945/1541) and 39.6\% (611/1541) of the multitype infections, respectively [18]. HPV 16 and 18 consistently showed relatively high co-detection rate.

\section{Summary}

HPV infection is considerably common among all kinds of populations. Multitype infection presents higher detection rates than single-type infections in all kinds of populations. This high detection rate may be due to the technical progress in detection methods, the variants of the virus, and the emergence of numerous new HPV strains [19]. Differences in the prevalence of HPV types are also noted. Generally, HPV 16 and 18 are the most common types in high-risk multitype infection, and HPV 6 is the most common in low-risk multitype infection. HPV 16 and 18 in high-risk multitype infection and HPV 6 in low-risk multitype infection may present higher coinfection rates than the other HPV types. However, whether this combination exerts synergistic action with other combination types or whether this high rate is related to its own pathogenicity needs further study.

Acknowledgments: None.

Conflict of interest: The author states no conflicts of interest.

\section{References}

[1] Cuzick J., Arbyn M., Sankaranarayanan R., Tsu V., Ronco G., Mayrand M.H., et al., Overview of human papillomavirus-based and other novel options for cervical cancer screening in developed and developing countries, Vaccine, 2008, 26(Suppl. 10), K29-K41.

[2] Fiqueiredo Alves R.R., Turchi M.D., Santos L.E., Guimarães E.M., Garcia M.M., Seixas M.S., et al., Prevalence, genotype profile and risk factors for multiple human papillomavirus cervical infection in unimmunized female adolescents in Goiania, Brazil: a community-based study, BMC Public Health, 2013, 13(1), 1041.

[3] Menegazzi P., Barzon L., Palù G., Reho E., Tagliaferro L., Human papillomavirus type distribution and correlation with cyto-histological patterns in women from the south of Italy, Infect. Dis. Obstet. Gynecol., 2009, 2009, 198425.

[4] Vaccarella S., Söderlund-Strand A., Franceschi S., Plummer M., Dillner J., Patterns of human papillomavirus types in multiple infections: an analysis in women and men of the high throughput human papillomavirus monitoring study, PLoS ONE, 2013, 8(8), e71617. 
[5] Chaturvedi A.K., Katki H.A., Hildesheim A., Rodríguez A.C., Quint W., Schiffman M., et al., Human Papillomavirus infection with multiple types: pattern of coinfection and risk of cervical disease, J. Infect. Dis., 2011, 203(7), 910-920.

[6] Gu Y., Zhou X., Wang J., Yu Q., Zang Y., Wang X., et al., The screening result and analysis of 12226 cases with HPV virus infection, Chin. Matern. Child Health Care, 2014, 29(32), 5208-5210.

[7] Dickson E.L., Vogel R.I., Bliss R.L., Downs L.S., Multi-type human papillomavirus (HPV) infections: a cross-sectional analysis of the prevalence of specific types in 309,000 women referred for HPV testing at the time of cervical cytology, Int. J. Gynecol. Cancer, 2013, 23(7), 1295-1302.

[8] Bayram A., Erkılıç S., Balat Ö, Ekşi F., Uğur M.G., Öztürk E., et al., Prevalence and genotype distribution of human papillomavirus in non-neoplastic cervical tissue lesion: cervical erosion, J. Med. Virol., 2011, 83(11), 1997-2003.

[9] Zhang J., Li X., Xu W., Fu S. HPV virus infection and subset analysis in Hainan, Hainan Med. J., 2015, 26(10), 1460-1463.

[10] Mollers M., Vriend H.J., van der Sande MA, van Bergen JE, King A.J., Lenselink C.H., et al., Population- and type-specific clustering of multiple HPV types across diverse risk populations in the Netherlands, Am. J. Epidemiol., 2014, 179(10), 1236-1246.

[11] Vidal A.C., Smith J.S., Valea F., Bentley R., Gradison M., Yarnall K.S., et al., HPV genotypes and cervical intraepithelial neoplasia in a multiethnic cohort in the southeastern USA, Cancer Causes Control, 2014, 25(8), 1055-1062.

[12] Spinillo A., Dal Bello B, Alberizzi P., Cesari S., Gardella B., Roccio M., et al., Clustering patterns of human papillomavirus genotypes in multiple infections, Virus Res., 2009, 142(1-2), 154-159.

[13] Beca F., Pinheiro J., Rios E., Pontes P., Amendoeira I., Genotypes and prevalence of HPV single and multiple concurrent infections in women with HSIL, Diagn. Cytopathol., 2014, 42(11), 919-923.

[14] Correnti M., Medina F., Cavazza M.E., Rennola A., Avila M., Fernándes A., Human papillomavirus (HPV) type distribution in cervical carcinoma, low-grade, and high-grade squamous intraepithelial lesions in Venezuelan women, Gynecol. Oncol., 2011, 121(3), 527-531.

[15] Denny L., Adewole I., Anorlu R., Dreyer G., Moodley M., Smith T., et al., Human papillomavirus prevalence and type distribution in invasive cervical cancer in sub-Saharan Africa, Int. J. Cancer, 2014, 134(6), 1389-1398.

[16] Sykes P., Gopala K., Tan A.L., Kenwright D., Petrich S., Molijn A., et al., Type distribution of human papillomavirus among adult women diagnosed with invasive cervical cancer (stage 1b or higher) in New Zealand, BMC Infect. Dis., 2014, $14,374$.

[17] Li D., Xiao Z., Chen Y., HPV epidemiological investigation of Cervical cancer patients in a certain area, Lab. Med. Clinic, 2014, 11(17), 2423-2424.

[18] Li N., Franceschi S., Howell-Jones R., Snijders P.J., Clifford G.M., Human papillomavirus type distribution in 30,848 invasive cervical cancers worldwide: variation by geographical region, histological type and year of publication, Int. J. Cancer, 2011, 128(4), 927-935.

[19] Bello B.D., Spinillo A., Alberizzi P., Cesari S., Gardella B., Silini E.M., Time trends of human papillomavirus type distribution in Italian women with cervical intraepithelial neoplasia (CIN), Gynecol. Oncol., 2009, 115(2), 262-266. 